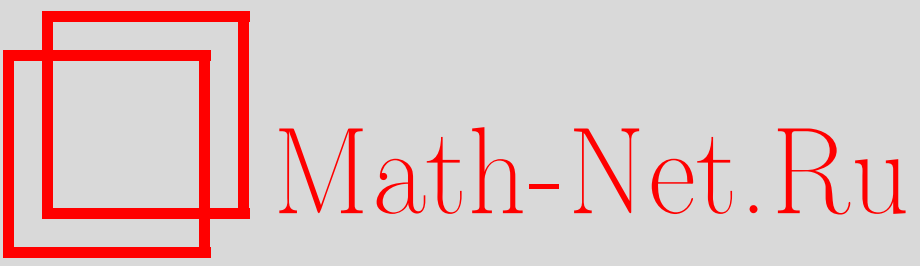

В. А. Матвеев, В. И. Саврин, А. Н. Сисакян, А. Н. Тавхелидзе, Релятивистские кварковые модели в квазипотенциальном подходе, ТМФ, 2002, том 132, номер 2, 267-287

DOI: https://doi.org/10.4213/tmf360

Использование Общероссийского математического портала Math-Net.Ru подразумевает, что вы прочитали и согласны с пользовательским соглашением

http://www.mathnet.ru/rus/agreement

Параметры загрузки:

IP: 34.227 .88 .159

26 апреля 2023 г., 12:58:10 
ТЕОРЕТИЧЕСКАЯ

И МАТЕМАТИЧЕСКАЯ

ФИЗИКА

Tом 132, № 2

август, 2002

(C) 2002 г

В.А. Матвеев*, В.И. Саврин ${ }^{\dagger}$, А.Н. Сисакян ${ }^{\ddagger}$, А.Н. Тавхелидзе*

\section{РЕЛЯТИВИСТСКИЕ КВАРКОВЫЕ МОДЕЛИ В КВАЗИПОТЕНЦИАЛЬНОМ ПОДХОДЕ}

В рамках квазипотенциального подхода описано взаимодействие релятивистских частиц. Изложение базируется на так называемой ковариантной одновременной формулировке квантовой теории поля, при которой теория рассматривается на пространственноподобной трехмерной гиперповерхности в пространстве Минковского. Особое внимание уделяется методам построения различных квазипотенциалов, а также приложениям квазипотенциального подхода к описанию характеристик взаимодействия релятивистских частиц в кварковых моделях: амплитуды упругого рассеяния адронов, спектров масс и ширин распадов мезонов, сечения глубоконеупругого рассеяния лептонов на адронах.

Ключевые слова: квазипотенциальное уравнение, релятивистские кварковые модели, упругое рассеяние адронов, формфакторы и спектры мезонов, структурные функции адронов.

\section{1. ВВЕДЕНИЕ}

Как показывают многочисленные эксперименты, большинство известных в настоящее время частиц обладают внутренней структурой, т.е. являются составными объектами. Это в первую очередь касается адронов, которые состоят из цветных кварков и глюонов. Однако уже сейчас интенсивно ведется экспериментальный поиск "экзотических" частиц, например, лептокварков и возбужденных лептонов, которые также могли бы иметь составную природу.

Описание спектров, вероятностей распадов и сечений рассеяния составных объектов требует построения последовательной теории связанных состояний. Такая теория должна базироваться на основных принципах локальной квантовой теории поля и использовать ее аппарат [1]. Однако непосредственное вычисление указанных характеристик составных систем в рамках локальной квантовой теории поля вряд ли всегда возможно, поскольку пока единственный известный способ расчетов в ней базируется на

\footnotetext{
* Институт ядерных исследований РАН, Москва, Россия

${ }^{\dagger}$ Научно-исследовательский институт ядерной физики Московского государственного университета, Москва, Россия

‡ Объединенный институт ядерных исследований, Дубна, Московская обл., Россия
} 
теории возмушений, в то время как природа образования связанных состояний взаимодействуюших частищ, безусловно, должна определяться непертурбативными эффектами.

Наиболее действенным способом выхода за рамки теории возмушений при построении теории связанных состояний является использование динамических уравнений. Дело в том, что даже если ядра (потенциалы) динамических уравнений удается построить только в низших порядках теории возмущений, разработка методов их точного или приближенного (но без использования теории возмушений) решения позволяет учесть вклад непертурбативных эффектов взаимодействия при вычислении наблюдаемых характеристик связанных состояний. В нерелятивистском случае подобная теория строится с помощью динамического уравнения Шредингера на языке классического потенциала. Однако при больших дефектах массы и высоких энергиях, которыми обладают ускоренные составные частицы, соответствующая теория должна быть существенно релятивистской. В связи с этим полвека назад наметился путь решения данной проблемы, основанный на использовании динамических уравнений в локальной квантовой теории поля, примерами которых могут служить уравнение Бете-Солпитера [2], квазипотенциальное уравнение [3] и др. [4].

В предлагаемой работе используется квазипотенциальный подход к описанию взаимодействия релятивистских частиц, который базируется на так называемой ковариантной одновременной формулировке квантовой теории поля [5], при которой эта теория строится на пространственноподобной трехмерной гиперповерхности в пространстве Минковского. Основной аргумент в пользу целесообразности использования такой формулировки состоит в том, что для расчета наблюдаемых характеристик процесса взаимодействия в системе частиц нет необходимости в описании протекания этого процесса во всем четырехмерном пространстве Минковского. Достаточно проследить эволюцию системы "на срезе", например, на пространственноподобной гиперплоскости. В этом случае с помощью преобразования Лоренца (поворота гиперплоскости) можно добиться совпадения времен всех частиц.

Поскольку, в отличие от четырехмерного формализма Бете-Солпитера, в квазипотенциальном подходе можно исключить относительные времена различных частиц в системе, не возникает необходимости формулировать граничные условия по этим переменным, что было бы практически невозможно сделать исходя из каких-либо физических соображений. Кроме того, наличие только одного единого времени при описании системы частиц позволяет использовать вероятностную интерпретацию квазипотенциальной волновой функции этой системы.

Таким образом, квазипотенциальный подход к описанию релятивистских составных систем базируется на принципах и методах локальной квантовой теории поля, но позволяет описывать системы взаимодействующих частиц на привычном языке волновой функции, удовлетворяющей релятивистскому трехмерному уравнению - аналогу уравнения Шредингера - и физическим граничным условиям. Последние фактически задаются введением процедуры проектирования и сглаживания в пределах выбранной пространственноподобной гиперповерхности.

Основная проблема квазипотенциального подхода состоит в выборе квазипотенциала взаимодействия. Поэтому в данной работе особое внимание уделяется методам построе- 
ния различных квазипотенциалов, а также приложениям квазипотенциального подхода к описанию характеристик взаимодействия релятивистских частиц в кварковых моделях: амплитуды упругого рассеяния адронов, спектров масс и ширин распадов мезонов, сечения глубоконеупругого рассеяния лептонов на адронах. Явная аналогия между описанием релятивистских систем с помощью трехмерного квазипотенциального уравнения и нерелятивистской картиной взаимодействия оказывается крайне полезной, поскольку позволяет использовать чисто эмпирические соображения классической физики при построении квазипотенциала взаимодействия.

\section{2. КВАЗИПОТЕНЦИАЛЬНОЕ УРАВНЕНИЕ И СВОЙСТВА КВАЗИПОТЕНЦИАЛА}

Рассмотрим волновую функцию Бете-Солпитера для системы фермиона и антифермиона [2]

$$
\Phi_{P}\left(x_{1}, x_{2}\right)=\left\langle 0\left|T\left\{\psi_{1}\left(x_{1}\right) \bar{\psi}_{2}\left(x_{2}\right) S\right\}\right| P\right\rangle,
$$

где $|P\rangle$ - вектор состояния из гильбертова пространства асимптотических состояний с определенным числом частиц и полным 4-импульсом $P$. Отметим, что это состояние может быть: а) одночастичным, когда речь идет о волновой функции связанного состояния; б) двухчастичным, и тогда мы имеем волновую функцию, отвечаюшую рассеянию; в) многочастичным, когда волновая функция (1) отвечает более сложному неупругому процессу.

В основе квазипотенциального подхода, или одновременной формулировки квантовой теории поля, лежит процедура сглаживания и проектирования волновой функции (1) с помошью одночастичных волновых функций фермиона и антифермиона $u^{(\mp)}(x ; k)$ на гиперплоскости $\lambda x=\tau$ в пространстве Минковского с времениподобным единичным 4-вектором нормали $\lambda\left(\lambda^{2}=1\right)$. В результате применения этой процедуры к волновой функции (1) получим волновую функцию в импульсном пространстве, зависяшую от единого инвариантного времени $\tau$,

$$
\begin{aligned}
& \Phi_{P}^{(-)}\left(\mathbf{k}_{1}, \mathbf{k}_{2} \mid \tau\right)= \\
& \quad=\int d^{4} x_{1} \delta\left(\lambda x_{1}-\tau\right) \int d^{4} x_{2} \delta\left(\lambda x_{2}-\tau\right) \bar{u}_{1}^{(+)}\left(k_{1} ; x_{1}\right) \hat{\lambda} \Phi_{P}\left(x_{1}, x_{2}\right) \hat{\lambda} u_{2}^{(+)}\left(x_{2} ; k_{2}\right)= \\
& \quad=(2 \pi)^{3} 2 k_{2}^{0} \delta^{(3)}\left[\mathbf{k}_{1}+\mathbf{k}_{2}-\mathbf{P}-\left(\varepsilon_{k_{1}}+\varepsilon_{k_{2}}-\varepsilon_{p}\right) \lambda\right] \exp \left\{i\left(\varepsilon_{k_{1}}+\varepsilon_{k_{2}}-\varepsilon_{p}\right) \tau\right\} \widetilde{\Phi}_{P}^{(-)}\left(\mathbf{k}_{1}\right),
\end{aligned}
$$

где $\varepsilon_{p}=(\lambda p)$ - инвариантная энергия, и введена стационарная волновая функция

$$
2 \varepsilon_{k_{2}} \widetilde{\Phi}_{P}^{(-)}\left(\mathbf{k}_{1}\right)=\int d^{4} x \delta(\lambda x) e^{i k_{1} x} \bar{v}_{1}^{(+)}\left(k_{1}\right) \hat{\lambda} \Phi_{P}(x, 0) \hat{\lambda} v_{2}^{(+)}\left(k_{2}\right),
$$

включающая в себя амплитуды одночастичных волновых функций $v^{(\mp)}(k)$.

Благодаря связи между трехмерными импульсами, которую накладывает $\delta$-функция в (2), и свойствам вектора $\lambda, 4$-импульсы удовлетворяют следуюшему соотношению:

$$
k_{1}+k_{2}-\left(\varepsilon_{k_{1}}+\varepsilon_{k_{2}}\right) \lambda=P-\varepsilon_{p} \lambda
$$


т.е. сохраняется не полный 4-импульс системы, а только его ортогональная к $\lambda$ составляюшая. Другая особенность волновой функции (2) состоит в том, что поскольку волновые функции фермиона и антифермиона удовлетворяют свободному уравнению Дирака, частицы всегда лежат на массовых гиперболоидах $k_{1}^{2}=m_{1}^{2}$ и $k_{2}^{2}=m_{2}^{2}$. Таким образом, проведенная процедура сглаживания и проектирования обеспечивает наличие единого для всей системы времени и исключает появление виртуальных частиц. Эти свойства позволяют использовать вероятностную интерпретацию волновой функции системы взаимодействующих релятивистских частищ и провести описание такой системы полностью в трехмерной форме.

Волновая функция (3) может описывать связанное состояние фермиона и антифермиона как со спином 1, так и со спином 0. Мы ограничимся здесь рассмотрением псевдоскалярного состояния с массой $M=\sqrt{P^{2}}$, для которого волновая функция имеет следующую структуру:

$$
\widetilde{\Phi}_{P}^{(-)}\left(\mathbf{k}_{1}\right)=\frac{\bar{v}_{1}^{(+)}\left(k_{1}\right) \gamma^{5} v_{2}^{(+)}\left(k_{2}\right)}{2 \varepsilon_{k_{2}}} \varphi_{P}\left(\mathbf{k}_{1}\right)
$$

где $\varphi_{P}\left(\mathbf{k}_{1}\right)$ - скалярная часть волновой функции, которая в случае $m_{1}=m_{2}=m$ удовлетворяет квазипотенциальному уравнению [5]

$$
2 \varepsilon_{k_{1}}\left(2 \varepsilon_{k_{1}}-M\right) \varphi_{P}\left(\mathbf{k}_{1}\right)=\frac{1}{m^{2}} \int d^{3} \omega_{\mathbf{k}_{1}^{\prime}}\left(2 \varepsilon_{k_{1}} \varepsilon_{k_{1}^{\prime}}-m^{2}\right) V\left(\mathbf{k}_{1} ; \mathbf{k}_{1}^{\prime} \mid P, M\right) \varphi_{P}\left(\mathbf{k}_{1}^{\prime}\right)
$$

с условием нормировки волновой функции

$$
\begin{aligned}
M=\int & d^{3} \omega_{\mathbf{k}_{1}} 2 \varepsilon_{k_{1}}\left|\varphi_{P}\left(\mathbf{k}_{1}\right)\right|^{2}+ \\
& +\frac{1}{m^{2}} \int d^{3} \omega_{\mathbf{k}_{1}} \int d^{3} \omega_{\mathbf{k}_{1}^{\prime}}\left(2 \varepsilon_{k_{1}} \varepsilon_{k_{1}^{\prime}}-m^{2}\right) \stackrel{\varphi}{\varphi}_{P}\left(\mathbf{k}_{1}\right) \frac{\partial}{\partial M} V\left(\mathbf{k}_{1} ; \mathbf{k}_{1}^{\prime} \mid P, M\right) \varphi_{P}\left(\mathbf{k}_{1}^{\prime}\right),
\end{aligned}
$$

где $d^{3} \omega_{\mathbf{k}}=d^{3} \mathbf{k} /(2 \pi)^{3} 2 k^{0}$ - инвариантный трехмерный элемент объема в импульсном пространстве на массовом гиперболоиде. В формулах (6) и (7) мы положили $\lambda=$ $P / \sqrt{P^{2}}$, т.е. направили $\lambda$ вдоль полного 4-импульса системы. Это соответствует так называемой калибровке Маркова-Юкавы, которая устраняет произвол в выборе направления $\lambda$ физически оправданным образом, поскольку единственным выделенным направлением в пространстве Минковского является полньй 4-импульс системы, которую мы изучаем. В этом случае

$$
k_{1}+k_{2}-\left(\varepsilon_{k_{1}}+\varepsilon_{k_{2}}\right) \lambda=k_{1}^{\prime}+k_{2}^{\prime}-\left(\varepsilon_{k_{1}^{\prime}}+\varepsilon_{k_{2}^{\prime}}\right) \lambda=0
$$

причем для равных масс фермионов $\varepsilon_{k_{1}}=\varepsilon_{k_{2}}$ и $\varepsilon_{k_{1}^{\prime}}=\varepsilon_{k_{2}^{\prime}}$.

Ядро трехмерного динамического уравнения (6) - квазипотеншиал $V\left(\mathbf{k}_{1} ; \mathbf{k}_{1}^{\prime} \mid P, \sqrt{s}\right)$, $s=P^{2}$ - представляет собой релятивистское обобшение квантово-механического потенциала, но в отличие от последнего является комплексным и нелокальным и зависит от энергии системы. Это отражает в первую очередь чисто релятивистские эффекты рождения частиц и запаздывания взаимодействия. Необходимость учета зависимости квазипотенциала от энергии, в частности, наглядно проявляется в условии нормировки (7), 
куда явно входит производная квазипотенциала по полной энергии. Важным свойством квазипотенциала, вытекающим из условия унитарности $S$-матрицы, является неотрицательность его усредненной мнимой части [6],

$$
\begin{aligned}
& \frac{1}{m^{2}} \int d^{3} \omega_{\mathbf{k}_{1}} \int d^{3} \omega_{\mathbf{k}_{1}^{\prime}}\left(2 \varepsilon_{k_{1}} \varepsilon_{k_{1}^{\prime}}-m^{2}\right) \stackrel{\varphi}{\varphi}_{P}\left(\mathbf{k}_{1}\right) \operatorname{Im} V\left(\mathbf{k}_{1} ; \mathbf{k}_{1}^{\prime} \mid P, \sqrt{s}\right) \varphi_{P}\left(\mathbf{k}_{1}^{\prime}\right)= \\
& =\frac{1}{2} H\left(\mathbf{p}_{1} ; \mathbf{p}_{1} \mid P\right) \geqslant 0,
\end{aligned}
$$

где $\varphi_{P}\left(\mathbf{k}_{1}\right)$ - волновая функция, отвечаюшая упругому рассеянию фермиона и антифермиона, причем $P=p_{1}+p_{2}$, а $H\left(\mathbf{p}_{1} ; \mathbf{p}_{1} \mid P\right)$ - диагональный вклад всех возможных неупругих каналов в условие унитарности. Кроме знакоопределенности, из формулы (9) следует, что наличие мнимой части у квазипотенциала связано с существованием процессов рождения и преврашения частиц в релятивистской физике.

\section{3. КВАЗИПОТЕНЦИАЛ ЭЛЕКТРОМАГНИТНОГО ВЗАИМОДЕЙСТВИЯ}

Для того чтобы можно было использовать интегральное уравнение (6) и условие нормировки (7) для нахождения собственных волновых функций и значений энергии, необходимо знать явньй вид квазипотенциала. Существуют различные методы построения квазипотенциала взаимодействия. Так, например, в случае слабой связи, когда применима теория возмущений, был найден регулярный способ построения локального квазипотенциала на основе метода двухвременных функций Грина, а также дисперсионных соотношений для амплитуд рассеяния по передаче импульса [7]. Указанный метод построения локального квазипотенциала был успешно применен для решения ряда задач электродинамики, в частности, при исследовании энергетических уровней позитрония и водородоподобных атомов [8], а также при изучении вопроса о сходимости разложений по константе связи в неперенормируемых теориях [9].

Было показано [8], [10], что квазипотенциал электромагнитного взаимодействия электрона с позитроном в псевдоскалярном состоянии в низшем приближении однофотонного обмена можно записать в виде

$$
V\left(\mathbf{k}_{1} ; \mathbf{k}_{1}^{\prime} \mid P, \sqrt{s}\right)=\frac{4 \pi \alpha(2 m)^{2}}{q\left(q-\sqrt{s}+\varepsilon_{k_{1}}+\varepsilon_{k_{1}^{\prime}}-i 0\right)},
$$

где $q=\sqrt{\left(\varepsilon_{k_{1}}-\varepsilon_{k_{1}^{\prime}}\right)^{2}-\left(k_{1}-k_{1}^{\prime}\right)^{2}}$. В системе покоя получаем

$$
\left.V\left(\mathbf{k} ; \mathbf{k}^{\prime} \mid P, \sqrt{s}\right)\right|_{\mathbf{P}=0}=\frac{4 \pi \alpha(2 m)^{2}}{\left|\mathbf{k}-\mathbf{k}^{\prime}\right|\left(\left|\mathbf{k}-\mathbf{k}^{\prime}\right|-\sqrt{s}+k^{0}+k^{\prime 0}-i 0\right)},
$$

где $k^{0}=\sqrt{\mathbf{k}^{2}+m^{2}}$. Этот квазипотенциал представляет собой релятивистское обобщение кулоновского потенщиала, полученное в низшем порядке теории возмущений в рамках квантовой электродинамики.

Обратим внимание на свойства квазипотенциала (11), которые отличают его от обычного кулоновского потенциала, используемого в классической физике или нерелятивистской квантовой механике для описания взаимодействия заряженных частиц. Во-первых, 
квазипотенциал (11) нелокален, т.е. зависит не только от разности импульсов $\mathbf{k}-\mathbf{k}^{\prime}$, но и непосредственно от них самих. Во-вторых, мы видим, что уже в низшем приближении квазипотенциал (11) в случае $\sqrt{s}>2 m$ имеет область, где его мнимая часть отлична от нуля,

$$
\left.\operatorname{Im} V\left(\mathbf{k} ; \mathbf{k}^{\prime} \mid P, \sqrt{s}\right)\right|_{\mathbf{P}=0}=(4 \pi m)^{2} \alpha \delta\left[\left(\mathbf{k}-\mathbf{k}^{\prime}\right)^{2}-\left(\sqrt{s}-k^{0}-k^{\prime 0}\right)^{2}\right] .
$$

Наконец, квазипотенциал (11) явно зависит от энергии рассматриваемой системы $\sqrt{s}$. Последнее обстоятельство существенно меняет постановку задачи на собственные значения для интегрального уравнения (6), поскольку в этом случае энергия связи $E=$ $M-2 m$ непосредственно входит в ядро этого уравнения.

В работе [10] было показано, что при достаточно малых импульсах частиц квазипотенциал (11) может быть приближенно сведен к локальному и исследован в конфигурационном пространстве относительных расстояний между частицами. При этом оказывается, что при $E>0$ квазипотенциал осциллирует, причем амплитуда осцилляций убывает по кулоновскому закону, а их частота равна $E /(\hbar c)$, т.е. обращается в нуль в нерелятивистском пределе $c \rightarrow \infty$. В случае пренебрежимо малого значения $E$ квазипотенциал переходит в обычный нерелятивистский кулоновский потенциал.

Отметим также, что картина рассеяния на осциллируюших квазипотенциалах, к тому же зависяших от энергии, может сушественно отличаться от привычной нам картины потенциального рассеяния, что, в частности, проявляется в сушествовании дискретных уровней, погруженных в непрерывный спектр [11].

\section{4. РЕЛЯТИВИСТСКОЕ КОНФИГУРАЦИОННОЕ ПРОСТРАНСТВО}

Квазипотенциальное уравнение (6) в системе покоя псевдоскалярного связанного состояния $\mathbf{P}=0$ принимает вид

$$
2 k^{0}\left(2 k^{0}-M\right) \varphi_{M}(\mathbf{k})=\frac{1}{m^{2}} \int d^{3} \omega_{\mathbf{k}^{\prime}}\left(2 k^{0} k^{\prime 0}-m^{2}\right) V\left(\mathbf{k} ; \mathbf{k}^{\prime} \mid P, M\right) \varphi_{M}\left(\mathbf{k}^{\prime}\right) .
$$

Переход к конфигурационному пространству с помошью обычного трехмерного преобразования Фурье для этого релятивистского уравнения, очевидно, теряет смысл, поскольку импульсное пространство в этом случае не евклидово (массовый гиперболоид). Поэтому в релятивистском случае для соответствующего преобразования были найдены функции, которые реализуют представление группы Лоренца как группы движений в пространстве Лобачевского на массовом гиперболоиде частицы [12],

$$
\xi(\mathbf{r} ; \mathbf{k})=\left(\frac{k n}{m}\right)^{-1-i m r},
$$

где $n=(1, \mathbf{r} / r)$ - светоподобный 4-вектор. Эти функции обладают необходимыми свойствами полноты и ортогональности. Разложение по ним квазипотенциала имеет вид

$$
V\left(\mathbf{k} ; \mathbf{k}^{\prime} \mid P, \sqrt{s}\right)=-(2 m)^{2} \int d^{3} \mathbf{r} \int d^{3} \mathbf{r}^{\prime} \stackrel{*}{\xi}(\mathbf{k} ; \mathbf{r}) V\left(\mathbf{r} ; \mathbf{r}^{\prime} \mid \sqrt{s}\right) \xi\left(\mathbf{r}^{\prime} ; \mathbf{k}^{\prime}\right) .
$$

Если теперь ввести локальный квазипотенциал

$$
V\left(\mathbf{r} ; \mathbf{r}^{\prime} \mid \sqrt{s}\right)=V(r \mid \sqrt{s}) \delta^{(3)}\left(\mathbf{r}-\mathbf{r}^{\prime}\right),
$$


где функция $V(r \mid \sqrt{s})$ представляет собой аналог локального центрально-симметричного потенциала в релятивистском конфигурационном пространстве и зависит от полной энергии состояния $\sqrt{s}$, то, подставляя (16) в формулу (15), получаем

$$
V\left(\mathbf{k} ; \mathbf{k}^{\prime} \mid P, \sqrt{s}\right)=V(\mathbf{q} \mid \sqrt{s})=-(2 m)^{2} \int d^{3} \mathbf{r} \stackrel{*}{\xi}(\mathbf{q} ; \mathbf{r}) V(r \mid \sqrt{s})
$$

где

$$
\mathbf{q}=\mathbf{k}(-) \mathbf{k}^{\prime} \equiv \mathbf{k}-\frac{\mathbf{k}^{\prime}}{m}\left(k^{0}-\frac{\left(\mathbf{k} \mathbf{k}^{\prime}\right)}{k^{\prime 0}+m}\right)
$$

- разность двух векторов в искривленном пространстве Лобачевского, реализованном на массовом гиперболоиде частицы. При этом обычный квадрат передачи 4-импульса записывается как

$$
t \equiv\left(k-k^{\prime}\right)^{2}=-2 m\left(\sqrt{\mathbf{q}^{2}+m^{2}}-m\right) .
$$

Для того чтобы записать квазипотенциальное уравнение (13) в релятивистском конфигурационном пространстве, необходимо построить в нем оператор свободного гамильтониана, удовлетворяющий уравнению

$$
\widehat{\mathcal{H}}_{\mathbf{r}}^{(0)} r \xi(\mathbf{r} ; \mathbf{k})=2 k^{0} r \xi(\mathbf{r} ; \mathbf{k}) .
$$

Этот оператор был найден. В сферических координатах он имеет следующий вид [12]:

$$
\widehat{\mathcal{H}}_{\mathbf{r}}^{(0)}=2 m\left[\operatorname{ch} i \lambda \frac{\partial}{\partial r}+\frac{\lambda^{2}}{2 r^{(2)}} \Delta_{\theta, \varphi} \exp \left(i \lambda \frac{\partial}{\partial r}\right)\right],
$$

где $r^{(2)}=r(r+i \lambda)$ и $\lambda=m^{-1}-$ комптоновская длина волны фермиона, т.е. оператор оказывается конечно-разностным. В результате квазипотенциальное уравнение в релятивистском конфигурационном пространстве принимает вид

$$
\widehat{\mathcal{H}}_{\mathbf{r}}^{(0)}\left(M-\widehat{\mathcal{H}}_{\mathbf{r}}^{(0)}\right) r \varphi_{M}(\mathbf{r})=\frac{1}{m} \widehat{\mathcal{H}}_{\mathbf{r}}^{(0)} V(r \mid \sqrt{s}) \widehat{\mathcal{H}}_{\mathbf{r}}^{(0)} r \varphi_{M}(\mathbf{r})-2 m V(r \mid \sqrt{s}) r \varphi_{M}(\mathbf{r}),
$$

где

$$
\varphi_{M}(\mathbf{r})=2 m \int d^{3} \omega_{\mathbf{k}} \xi(\mathbf{r} ; \mathbf{k}) \varphi_{M}(\mathbf{k})
$$

Нетрудно показать, что в нерелятивистском пределе, когда $\lambda \rightarrow 0$, уравнение $(21)$ переходит в дифференциальное уравнение Шредингера.

Ясно, что появление конечно-разностных операторов в уравнении (21) связано с чисто релятивистскими эффектами - законом дисперсии (связь энергии и импульса частицы) и нелокальностью ядра квазипотенциального уравнения (13). В свое время необходимость введения конечно-разностных операций породила эвристическую идею о квантовании релятивистского конфигурационного пространства. Однако эта идея не получила дальнейшего глубокого развития.

Разложим волновую функцию (22) по парциальным волнам,

$$
r \varphi_{M}(\mathbf{r})=\sum_{l=0}^{\infty}(2 l+1) \varphi_{M}^{(l)}(r) P_{l}\left(\cos \theta_{\mathbf{r}}\right) .
$$

4 Теоретическая и математическая физика, т. 132, № 2, 2002 г. 
То же самое можно сделать с волновой функцией в импульсном пространстве,

$$
k \varphi_{M}(\mathbf{k})=4 \pi \sum_{l=0}^{\infty}(2 l+1) P_{l}\left(\cos \theta_{\mathbf{k}}\right) \varphi_{M}^{(l)}(\chi) .
$$

Мы ввели здесь переменную быстроты $\chi=\ln \left(k^{0}+k\right) / m$, которую удобно использовать для параметризации энергии и импульса частицы на массовом гиперболоиде, поскольку $k^{0}=m \operatorname{ch} \chi$ и $k=m \operatorname{sh} \chi$. Кроме того, именно эта переменная $\chi$, а не импульс $k$, сопряжена с релятивистской координатой $r$, что наиболее явно следует из связи парциальных волновых функций S-состояния,

$$
\varphi_{M}^{(0)}(\chi)=\int_{0}^{\infty} d r \sin (m r \chi) \varphi_{M}^{(0)}(r) .
$$

\section{5. КВАЗИПОТЕНЦИАЛ СИЛЬНОГО ВЗАИМОДЕЙСТВИЯ И УПРУГОЕ РАССЕЯНИЕ АДРОНОВ}

В разделе 3 мы исследовали свойства квазипотенциала взаимодействия заряженных частиц (10), построенного в низшем порядке теории возмушений в рамках квантовой электродинамики. Однако такое построение возможно только в тех случаях, когда константа связи мала. Если же мы хотим описать взаимодействие адронов, такой подход будет заведомо некорректным, поскольку в этом случае мы имеем дело с сильным взаимодействием. Поэтому квазипотенциальньй подход к описанию рассеяния адронов при высоких энергиях основывался на феноменологическом выборе локального двухчастичного квазипотенциала (16). При этом, конечно, следует опираться на общие принципы локальной теории поля, такие, как релятивистская инвариантность, аналитичность, унитарность и перекрестная симметрия. Уже в первых работах по квазипотенциальному подходу в квантовой теории поля было показано, как исходя из дисперсионных соотношений по передаче импульса можно построить локальный квазипотенциал в виде непрерывной суперпозиции потенциалов Юкавы с зависяшей от энергии плотностью [7],

$$
V(\mathbf{q} \mid \sqrt{s})=\int_{4 m^{2}}^{\infty} d \mu^{2} \frac{\sigma\left(\mu^{2} \mid \sqrt{s}\right)}{\mu^{2}-t} .
$$

Квазипотенциалы такого типа были использованы при изучении асимптотического поведения амплитуд в квантовой теории поля. С их помощью, в частности, была исследована природа реджевского асимптотического поведения амплитуды двухчастичного рассеяния и отмечена возможность появления точек ветвления парциальных амплитуд в комплексной плоскости углового момента [9].

При построении квазипотенциала, кроме указанных общих ограничений, используются также эмпирические закономерности двухчастичного рассеяния при высоких энергиях, наблюдаемые в эксперименте. При этом весьма плодотворной оказалась гипотеза о гладкости локального квазипотенциала, выдвинутая в работе [13]. Свойство гладкости локального квазипотенциала отражает важное динамическое свойство двухчастичных взаимодействий при высоких энергиях и, образно говоря, означает, что в высокоэнергетических столкновениях адроны ведут себя как "рыхлые" протяженные объекты 
конечных размеров. Эта гипотеза позволила объяснить целый ряд основных закономерностей упругого рассеяния частиц высоких энергий на малые и большие углы [14]. В частности, для гладких потенциалов удалось доказать справедливость эйконального приближения для амплитуды рассеяния, которое правильно воспроизводит наблюдаемую экспериментально дифракционную картину упругого рассеяния на малые углы и экспоненциальное падение дифференциальных сечений с ростом переданного импульса.

Квазипотенциальное уравнение для амплитуды упругого рассеяния двух одинаковых частиц (например, протонов) в системе центра масс $\mathbf{P}=0$ имеет вид

$$
T(\mathbf{k} ; \mathbf{p} \mid P, \sqrt{s})=V(\mathbf{k} ; \mathbf{p} \mid P, \sqrt{s})+\int d^{3} \omega_{\mathbf{k}^{\prime}} \frac{V\left(\mathbf{k} ; \mathbf{k}^{\prime} \mid P, \sqrt{s}\right) T\left(\mathbf{k}^{\prime} ; \mathbf{p} \mid P, \sqrt{s}\right)}{2 k^{\prime 0}\left(2 k^{\prime 0}-\sqrt{s}-i 0\right)},
$$

причем амплитуда $T(\mathbf{k} ; \mathbf{p} \mid P, \sqrt{s})$ находится вне энергетической поверхности, т.е. $2 k^{0} \neq$ $2 p^{0} \neq \sqrt{s}$. Решение этого уравнения в области высоких энергий и ограниченных переданных импульсов, $|t| / s \ll 1$, можно искать путем итераций. В первом борновском приближении амплитуда, очевидно, просто совпадает с квазипотенциалом. Учитывая, что наблюдаемое дифференшиальное сечение рассеяния в области малых передач имеет форму дифракционного пика, мы, пренебрегая спинами частищ, запишем локальный квазипотенциал в виде

$$
V(\mathbf{q} \mid \sqrt{s})=i s g \exp (a t)=i s g \exp \left[-2 m a\left(\sqrt{\mathbf{q}^{2}+m^{2}}-m\right)\right] .
$$

В силу (17) в конфигурационном пространстве будем иметь

$$
V(r \mid \sqrt{s})=-\frac{1}{2 m} \int d^{3} \omega_{\mathbf{q}} \xi(\mathbf{r} ; \mathbf{q}) V(\mathbf{q} \mid \sqrt{s})=-\frac{i s g}{(4 \pi)^{2} a m} e^{2 m^{2} a} K_{i m r}\left(2 m^{2} a\right) .
$$

Используя теперь выражение (28), вторую итерацию уравнения (27) на энергетической поверхности можно записать в виде

$$
\begin{aligned}
& \int d^{3} \omega_{\mathbf{k}^{\prime}} \frac{V\left(\mathbf{k} ; \mathbf{k}^{\prime} \mid P, \sqrt{s}\right) V\left(\mathbf{k}^{\prime} ; \mathbf{p} \mid P, \sqrt{s}\right)}{2 k^{\prime 0}\left(2 k^{\prime 0}-\sqrt{s}-i 0\right)}= \\
& \quad=(i s g)^{2} \exp \left(\frac{a t}{2}\right) \int d^{3} \omega_{\mathbf{k}^{\prime}} \frac{\exp \left[2 a\left(k^{\prime 0}-p^{0}\right)^{2}-2 a\left(\mathbf{k}^{\prime}-\mathbf{l}\right)^{2}\right]}{2 k^{\prime 0}\left(2 k^{\prime 0}-\sqrt{s}-i 0\right)},
\end{aligned}
$$

где $\mathbf{l}=(\mathbf{k}+\mathbf{p}) / 2$. Интегрируя по углам в сферических координатах, получаем

$$
\frac{(i s g)^{2}}{(4 \pi)^{2} a l} \exp \left(\frac{a t}{2}\right) \int_{-\infty}^{\infty} d k^{\prime} k^{\prime} \frac{\exp \left[2 a\left(k^{\prime 0}-p^{0}\right)^{2}-2 a\left(k^{\prime}-l\right)^{2}\right]}{\left(2 k^{\prime 0}\right)^{2}\left(2 k^{\prime 0}-\sqrt{s}-i 0\right)}
$$

где $2 l=\sqrt{s-4 m^{2}+t}=\sqrt{-u}$ и $2 p=\sqrt{s-4 m^{2}}$. В результате мнимая часть амплитуды в приближении двух итераций и в области ограниченных передач импульса $|t| / s \ll 1$ имеет вид

$$
\operatorname{Im} T(\mathbf{k} ; \mathbf{p} \mid P, \sqrt{s}) \simeq s g\left[\exp (a t)-\frac{g}{32 \pi a} \exp \left(\frac{a t}{2}\right)+\cdots\right] .
$$


Отметим, что линейному росту реджевской траектории померона будет отвечать следуюшая зависимость параметра $a$ от энергии столкновения:

$$
a(s)=a_{0}+\alpha^{\prime} \ln \frac{s}{s_{0}} .
$$

Из разложения (32) вытекают условия применимости второго борновского приближения,

$$
\frac{g}{32 \pi a} \ll 1, \quad a|t| \leqslant 1 .
$$

Ясно, что при достаточно быстро растушем полном сечении первое из этих условий может не выполняться. Тогда, по-прежнему, при $|t| / s \ll 1$ решение квазипотенциального уравнения (27) для амплитуды рассеяния может быть найдено в виде бесконечного ряда борновских приближений,

$$
\operatorname{Im} T(\mathbf{k} ; \mathbf{p} \mid P, \sqrt{s})=-8 \pi a s \sum_{n=1}^{\infty} \frac{\exp \left(\frac{a t}{n}\right)}{n n !}\left(-\frac{g}{8 \pi a}\right)^{n} .
$$

Этот ряд является абсолютно сходяшимся при любых фиксированных передачах импульса и, следовательно, определяет целую аналитическую функцию переменной $t$. Отметим, что знакопеременность борновского ряда приводит к появлению дифракционных минимумов в дифференциальном сечении, наблюдаемых в экспериментах по протон-протонному рассеянию при умеренных передачах импульса.

Суммируя ряд (35) при $t=0$ и используя оптическую теорему, получаем

$$
\sigma_{\text {tot }}(s)=8 \pi a\left[\ln \frac{g}{8 \pi a}+C-\operatorname{Ei}\left(-\frac{g}{8 \pi a}\right)\right] .
$$

В результате при $g /(8 \pi a) \rightarrow \infty$ асимптотика полного сечения имеет вид

$$
\sigma_{\text {tot }}(s) \simeq 8 \pi a \ln \frac{g}{8 \pi a} .
$$

При степенном росте функции $g(s)$ и с учетом зависимости (33) эта асимптотика совпадает с абсолютным фруассаровским ограничением $\sigma_{\text {tot }}(s) \sim \ln ^{2}(s / \bar{s})$.

Нетрудно видеть, что с ростом $|t|$ всё более старшие члены ряда (35) дают основной вклад в амплитуду рассеяния. При $a|t| \gg 1$ сумма ряда (35) может быть оценена с помошью метода перевала,

$$
\operatorname{Im} T(\mathbf{k} ; \mathbf{p} \mid P, \sqrt{s}) \simeq-\frac{8 \pi a s}{\sqrt{2 a|t|}} \exp (-\sqrt{2 a|t| \ln 2 a|t|}) .
$$

Таким образом, при больших $t$ дифференциальное сечение становится более пологим, а его осцилляции сглаживаются.

При описании упругого рассеяния при высоких энергиях часто используется так называемое эйкональное приближение. Оно основано на квазиклассической картине высокоэнергетического рассеяния, когда длины волн сталкиваюшихся частиц гораздо меньше эффективных размеров области взаимодействия. Эйкональное представление для 
амплитуды рассеяния легко вывести, используя решение квазипотенциального уравнения в форме итераций для гладких квазипотенциалов (35).

Подставляя

$$
\frac{\exp \left(\frac{a t}{n}\right)}{n}=\frac{1}{4 \pi a} \int d^{2} \boldsymbol{\rho} \exp \left\{i \boldsymbol{\rho} \boldsymbol{\Delta}_{T}-\frac{n \boldsymbol{\rho}^{2}}{4 a}\right\}
$$

(где $\boldsymbol{\rho}$ - двумерный вектор прицельного параметра, $t=-\boldsymbol{\Delta}_{T}^{2}$ и $\boldsymbol{\Delta}_{T}=\mathbf{p}-\mathbf{k}$ меняется в плоскости, перпендикулярной вектору l) в ряд (35), получаем

$$
T(\mathbf{k} ; \mathbf{p} \mid P, \sqrt{s})=4 s \int d^{2} \boldsymbol{\rho} e^{i \boldsymbol{\rho} \boldsymbol{\Delta}_{T}}\left(\frac{\exp [2 i \delta(\rho)]-1}{2 i}\right),
$$

где

$$
\delta(\rho)=\frac{i g}{16 \pi a} \exp \left(-\frac{\rho^{2}}{4 a}\right)
$$

Фазовая функция $\delta(\rho)$ (эйконал) связана с квазипотенциалом соотношением

$$
\delta(\rho)=\frac{m^{2}}{s} \int_{-\infty}^{+\infty} d z V\left(\sqrt{\rho^{2}+z^{2}} \mid \sqrt{s}\right)
$$

которое имеет место для широкого класса гладких квазипотенциалов [14], [15].

\section{6. ОПИСАНИЕ СПЕКТРОВ МЕЗОНОВ}

В квазипотенциальном подходе адроны (по аналогии с нерелятивистской теорией) рассматриваются как связанные состояния релятивистских кварков, взаимодействие между которыми описывается квазипотенциалами, допускающими локализованные в пространстве решения квазипотенциального уравнения при некоторых дискретных значениях энергии. Следует заметить, что используемый обычно вывод квазипотенциального уравнения в случае взаимодействия кварков может носить, вообще говоря, только чисто формальный характер, поскольку в силу конфайнмента кварки не обладают физическими асимптотическими состояниями. Однако если рассматривать этот вывод лишь как некоторую математическую процедуру, то она оказывается вполне корректной и приводит к физической картине, аналогичной той, которую мы имеем в нерелятивистской квантовой механике с бесконечно растущим потенциалом. Другими словами, мы как бы допускаем, что асимптотические состояния существуют, но в бесконечно удаленной области, влияние которой пока никак не сказывается на реально происходящих процессах.

Нерелятивистская потенциальная теория, базирующаяся на уравнении Шредингера, давно и достаточно широко применялась для описания мезонов как связанных состояний кварка и антикварка (см., например, [16]). Однако, как мы увидим ниже, спектры масс и ширины распадов мезонов, полученные в квазипотенциальном подходе, могут сильно отличаться от квантово-механических, т.е. учет релятивизма во многих случаях может оказаться сушественным. 
При описании связанных кварков мы снова имеем дело с сильным взаимодействием, поэтому при восстановлении квазипотенциала нельзя воспользоваться теорией возмущений. В этом случае для построения квазипотенциала кварк-кваркового взаимодействия можно пойти несколько эвристическим путем. Прежде всего, будем опираться на аналогию квазипотенциала и обычного потенциала, а также явную схожесть трехмерного релятивистского описания взаимодействия, основанного на квазипотенциальном уравнении, с описанием взаимодействия в нерелятивистской квантовой механике.

В теории сильных взаимодействий - квантовой хромодинамике (КХД) - взаимодействие между кварками осушествляется путем обмена безмассовым глюоном, и поэтому естественно предположить, что квазипотенциал $V(r \mid \sqrt{s})$ в релятивистском конфигурационном пространстве имеет кулоновский вид,

$$
V(r \mid \sqrt{s})=-\frac{\alpha_{s}}{r},
$$

где константа связи, вообще говоря, может зависеть от энергии. Подставляя этот квазипотенциал в преобразование (17), получаем в импульсном пространстве [17], что

$$
V(\mathbf{q} \mid \sqrt{s})=\frac{16 \pi \alpha_{s} m}{|\mathbf{q}| \ln \left(\sqrt{1+\frac{\mathbf{q}^{2}}{m^{2}}}+\frac{|\mathbf{q}|}{m}\right)} .
$$

Рассмотрим асимптотику поведения квазипотенциала в области малых расстояний или при $Q^{2} \rightarrow \infty$,

$$
V(\mathbf{q} \mid \sqrt{s}) \simeq \frac{32 \pi \alpha_{s}}{\frac{Q^{2}}{m^{2}} \ln \frac{Q^{2}}{m^{2}}} .
$$

Мы видим, что асимптотика квазипотенциала в этом случае согласуется с известным асимптотически свободным поведением одноглюонного вклада в КХД. При этом мы не делали никаких предположений о зависимости $\alpha_{s}$ от передачи импульса, т.е. в данном подходе изначально не возникает необходимости введения "бегушей" константы связи. Оказывается, что роль хорошо известной в КХД масштабной константы здесь играет масса конституэнтного кварка. Для простоты мы не рассматривали здесь цветовых степеней свободы, присущих КХД, однако можно показать, что их введение не изменило бы качественно вывод о том, что КХД взаимодействие на малых расстояниях воспроизводится обычным кулоновским потенциалом, но в релятивистском конфигурационном пространстве.

Расчет спектра связанных состояний в релятивистском кулоновском потенциале (43) с использованием уравнения (21) относится к классу точно решаемых задач [12]. В случаe S-состояния спектр масс радиальных возбуждений связанного состояния имеет вид

$$
M_{n}^{2}=2 m^{2}\left(1+\frac{1}{\sqrt{1+\frac{\alpha_{s}^{2}}{n^{2}}}}\right) .
$$

Очевидно, что в случае малой константы связи $\alpha_{s}$ энергия связи должна быть мала $\left(M_{n} \rightarrow 2 m\right)$ и спектр (46) становится близким к обычному нерелятивистскому кулоновскому спектру

$$
E_{n}=-\frac{\alpha_{s}^{2} m}{4 n^{2}} .
$$


В случае же сильных полей релятивистский спектр (46) существенно отличается от кулоновского и становится линейно растущим для низколежаших уровней $\left(n \ll \alpha_{s}\right)$,

$$
E_{n}=-(2-\sqrt{2}) m+\frac{n m}{\sqrt{2} \alpha_{s}}
$$

в то время как для $n \gg \alpha_{s}$ он будет близок к нерелятивистскому спектру (47). Хотелось бы обратить внимание на важную особенность этого спектра, заключающуюся в том, что даже в очень сильных полях энергия связи основного состояния не может быть больше чем $(2-\sqrt{2}) m$. По-видимому, это связано с тем, что вьше этого порога в сильных полях должно происходить рождение пар новых частиц из вакуума и, следовательно, такое состояние уже нельзя описывать как двухчастичную систему, что мы предполагали при постановке задачи.

Конечно, для описания реальных спектров мезонов, представляемых как связанные состояния кварков и антикварков, необходимо вводить “запираюший” потенциал, препятствуюший их вылетанию. Как было показано в ряде работ, хорошие результаты при описании экспериментальных данных по спектрам масс и ширинам распадов мезонов получаются при использовании комбинированных потенциалов типа "воронки”, например,

$$
V(r \mid \sqrt{s})=-\frac{\alpha_{s}}{r}+\sigma r .
$$

Получить точное решение трехмерного квазипотенциального уравнения (21) в этом случае не удается, поэтому здесь был применен метод квазиклассического приближения [18]. Для наглядности рассмотрим случай S-волны. Тогда квазипотенциальное уравнение $(21)$ для парциальной волны с $l=0$ можно записать в виде

$$
\operatorname{ch} i \lambda \frac{\partial}{\partial r}\left(a-\operatorname{ch} i \lambda \frac{\partial}{\partial r}\right) \varphi_{M}^{(0)}(r)=2 \operatorname{ch} i \lambda \frac{\partial}{\partial r} v(r) \operatorname{ch} i \lambda \frac{\partial}{\partial r} \varphi_{M}^{(0)}(r)-v(r) \varphi_{M}^{(0)}(r),
$$

где $a=M / 2 m$ и $v(r)=V(r \mid M) / 2 m$.

В квазиклассическом приближении решение уравнения (21) для парциальной волновой функции $\varphi_{M}^{(0)}(r)$ ишется в виде

$$
\varphi_{M}^{(0)}(r) \sim \exp \left[\frac{i}{\lambda} g(r)\right]
$$

где $g(r)$ разлагается в ряд

$$
g(r)=g_{0}(r)+\frac{\lambda}{i} g_{1}(r)+\left(\frac{\lambda}{i}\right)^{2} g_{2}(r)+\cdots
$$

Подставляя это разложение в уравнение (21) и приравнивая коэффициенты при одинаковых степенях $\lambda$, получаем для первых двух членов разложения

$$
\begin{gathered}
g_{0}^{\prime}(r)= \pm \chi(r), \\
g_{1}(r)=-\frac{1}{2} \ln \operatorname{sh} \chi(r)\{2[2 v(r)+1] \operatorname{ch} \chi(r)-a\},
\end{gathered}
$$


где

$$
\operatorname{ch} \chi(r)=\frac{\sqrt{4 v(r)[2 v(r)+1]+a^{2}}+a}{2[2 v(r)+1]} .
$$

Очевидно, классическая точка поворота $r_{+}$будет определяться соотношением $\operatorname{ch} \chi\left(r_{+}\right)=1$, что, как нетрудно показать, эквивалентно равенству $v\left(r_{+}\right)=a-1$. Мы рассмотрим здесь случай $a>1$. Квазиклассическое решение квазипотенциального уравнения в классически доступной области слева от этой точки поворота будет иметь вид

$$
\varphi_{M}^{(0)}(r)=\frac{C}{\sqrt{\operatorname{sh} \chi(r)\{2[2 v(r)+1] \operatorname{ch} \chi(r)-a\}}} \sin \left[\frac{1}{\lambda} \int_{r}^{r_{+}} \chi(r)+\frac{\pi}{4}\right] .
$$

Другая точка поворота $r_{-}<r_{+}$будет определяться равенством $2 v\left(r_{-}\right)+1=0$, где $\operatorname{ch} \chi(r)$ обрашается в бесконечность, а волновая функция должна обратиться в нуль. Следовательно, решение справа от этой точки поворота должно иметь вид

$$
\varphi_{M}^{(0)}(r)=\frac{C^{\prime}}{\sqrt{\operatorname{sh} \chi(r)\{2[2 v(r)+1] \operatorname{ch} \chi(r)-a\}}} \sin \left[\frac{1}{\lambda} \int_{r_{-}}^{r} \chi(r)\right] .
$$

Таким образом, квазиклассическое условие квантования задается формулой

$$
\int_{r_{-}}^{r_{+}} \chi(r)=\pi\left(n+\frac{3}{4}\right) \lambda
$$

Точка поворота $r_{-}$имеет чисто релятивистское происхождение, и при умеренных константах в используемом нами приближении $r_{-} \simeq \alpha_{s} \lambda$.

Отметим, что введенная здесь функция $\chi(r)$ имеет смысл быстроты частицы, движущейся в поле $v(r)$. Таким образом, как и при рассмотрении в импульсном пространстве, мы приходим к вьводу, что в релятивистском случае наиболее естественным оказывается описание в терминах быстроты, которая является непосредственным обобшением нерелятивистского импульса $p(r)=m \sqrt{a-1-v(r)}$ в квазиклассическом подходе.

Квазиклассическое приближение для квазипотенциального уравнения в релятивистском конфигурационном пространстве позволяет достаточно удовлетворительно рассчитать спектры масс и ширины распадов мезонов (см., например, [19]).

\section{7. ФОРМФАКТОРЫ РАСПАДА МЕЗОНОВ}

В квазипотенциальном подходе амплитуда распада мезона, состоящего из кварка и антикварка и описываемого волновой функцией $\widetilde{\Phi}_{P}^{(-)}$, на две частицы записывается следуюшим образом:

$$
\begin{aligned}
\mathcal{M}\left(\mathbf{p}_{1} \mid P\right)= & \int d^{3} \omega_{\mathbf{k}_{1}} T\left(\mathbf{p}_{1} ; \mathbf{k}_{1} \mid P, M\right) \int d^{3} \omega_{\mathbf{k}_{1}^{\prime}} \widetilde{G}^{(0)}\left(\mathbf{k}_{1} ; \mathbf{k}_{1}^{\prime} \mid P, M\right) \times \\
& \times \int d^{3} \omega_{\mathbf{k}_{1}^{\prime \prime}}\left[\widetilde{G}^{(-)}\right]^{-1}\left(\mathbf{k}_{1}^{\prime} ; \mathbf{k}_{1}^{\prime \prime} \mid P, M\right) \widetilde{\Phi}_{P}^{(-)}\left(\mathbf{k}_{1}^{\prime \prime}\right),
\end{aligned}
$$


где $\mathbf{p}_{1}$ - импульс одного из продуктов распада, $\widetilde{G}^{(0)}(P, M)$ и $\widetilde{G}^{(-)}(P, M)$ - функции Грина системы свободных и взаимодействуюших кварка и антикварка, $T(P, M)$ - амплитуда перехода системы кварк-антикварк в две частицы, на которые распадается мезон. Если пренебречь взаимодействием кварка и антикварка в промежуточном состоянии (поправками КХД), то [20]

$$
\mathcal{M}\left(\mathbf{p}_{1} \mid P\right)=\int d^{3} \omega_{\mathbf{k}_{1}} T\left(\mathbf{p}_{1} ; \mathbf{k}_{1} \mid P, M\right) \widetilde{\Phi}_{P}^{(-)}\left(\mathbf{k}_{1}\right)
$$

Рассмотрим распадзаряженного псевдоскалярного мезона на лептон-антилептонную пару, например, $\pi^{-} \rightarrow e \bar{\nu}_{e}$. Исходя из лагранжиана стандартной модели можно вычислить амплитуду $T(P, M)$ в низшем порядке по слабой константе связи,

$$
\begin{aligned}
T\left(\mathbf{p}_{1} ; \mathbf{k}_{1} \mid P, M\right)= & \frac{G}{\sqrt{2}\left(M_{W}^{2}-M^{2}\right)} \bar{v}_{e}^{(+)}\left(p_{1}\right) \gamma^{\mu}\left(1-\gamma^{5}\right) v_{\nu}^{(+)}\left(p_{2}\right) \times \\
& \times\left(M_{W}^{2} g_{\mu \nu}-P_{\mu} P_{\nu}\right) \bar{v}_{d}^{(-)}\left(k_{2}\right) \gamma^{\nu}\left(1-\gamma^{5}\right) v_{u}^{(-)}\left(k_{1}\right),
\end{aligned}
$$

где $G / \sqrt{2}=g^{2} /\left(8 M_{W}^{2}\right)$. Подставляя выражения (61) и (5) в (60), получим

$$
\mathcal{M}\left(\mathbf{p}_{1} \mid P\right)=\frac{G}{\sqrt{2}} f_{\pi} \bar{v}_{e}^{(+)}\left(p_{1}\right) \widehat{P}\left(1-\gamma^{5}\right) \gamma^{5} v_{\nu}^{(+)}\left(p_{2}\right),
$$

где константа распада выражается через волновую функцию,

$$
f_{\pi}=\frac{4 m_{q}}{M} \int d^{3} \omega_{\mathbf{k}_{1}} \varphi_{M}^{\pi}\left(\mathbf{k}_{1}\right)
$$

Мы положили здесь $m_{u}=m_{d}=m_{q}$. Нетрудно вычислить теперь ширину распада

$$
\Gamma_{\pi^{-} \rightarrow e \bar{\nu}_{e}}=\frac{3 G^{2} M m_{e}^{2}}{8 \pi} f_{\pi}^{2}\left(1-\frac{m_{e}^{2}}{M^{2}}\right)^{2},
$$

где $m_{e}$ - масса электрона и множитель 3 - цветовой фактор.

Рассмотрим теперь распад векторного мезона на электрон-позитронную пару. Для этого снова воспользуемся формулой (60), однако вместо (5) в этом случае для квазипотенциальной волновой функции следует применить формулу

$$
\widetilde{\Phi}_{P}^{(-)}\left(\mathbf{k}_{1}\right)=\frac{\bar{v}_{q}^{(+)}\left(k_{1}\right) \hat{e} v_{q}^{(+)}\left(k_{2}\right)}{2 \varepsilon_{k_{2}}} \varphi_{P}^{V}\left(\mathbf{k}_{1}\right),
$$

где $e^{\mu}-4$-вектор поляризации векторного мезона, удовлетворяюший условиям $(P e)=0$ и $(\stackrel{*}{e} e)=-1$. Исходя из лагранжиана квантовой электродинамики можно вычислить амплитуду аннигиляции кварк-антикварковой пары в электрон-позитронную в низшем порядке теории возмушений,

$$
T\left(\mathbf{p}_{1} ; \mathbf{k}_{1} \mid P, M\right)=-\frac{4 \pi \alpha e_{q}}{M^{2}} \bar{v}_{e}^{(+)}\left(p_{1}\right) \gamma_{\mu} v_{e}^{(+)}\left(p_{2}\right) \bar{v}_{q}^{(-)}\left(k_{2}\right) \gamma^{\mu} v_{q}^{(-)}\left(k_{1}\right),
$$


где $e_{q}-$ заряд кварка. Подставляя эту амплитуду и волновую функцию (65) в формулу (60), получаем

$$
\mathcal{M}\left(\mathbf{p}_{1} \mid P\right)=4 \pi \alpha f_{V} \bar{v}_{e}^{(+)}\left(p_{1}\right) \hat{e} v_{e}^{(+)}\left(p_{2}\right),
$$

где формфактор распада $V \rightarrow e^{-} e^{+}$в системе покоя

$$
f_{V}=\frac{2 e_{q}}{(2 \pi)^{3} M^{2}} \int d^{3} \mathbf{k}_{1}\left[1-\frac{\left(\mathbf{e k}_{1}\right)^{2}}{\mathbf{k}_{1}^{2}+m_{q}^{2}}\right] \varphi_{M}^{V}\left(\mathbf{k}_{1}\right) .
$$

При этом ширина распада

$$
\Gamma_{V \rightarrow e^{-} e^{+}}=4 \pi \alpha^{2} M f_{V}^{2}\left(1-\frac{4 m_{e}^{2}}{M^{2}}\right)^{1 / 2}\left(1+\frac{2 m_{e}^{2}}{M^{2}}\right) .
$$

Рассмотрим так называемый статический предел, когда внутренним движением кварков в мезоне можно пренебречь. В этом случае формула (68) принимает вид

$$
f_{V}=\frac{2 e_{q}}{(2 \pi)^{3} M^{2}} \int d^{3} \mathbf{k}_{1} \varphi_{M}^{V}\left(\mathbf{k}_{1}\right)=\frac{2 e_{q}}{M^{3 / 2}} \widetilde{\varphi}_{M}^{V}(0),
$$

где $\widetilde{\varphi}_{M}^{V}(\mathbf{r})$ - волновая функция в координатном пространстве, нормированная на единицу. Подставляя это выражение в формулу для ширины (69), получаем

$$
\Gamma_{V \rightarrow e^{-} e^{+}}=\frac{16 \pi \alpha^{2} e_{q}^{2}}{M^{2}}\left|\widetilde{\varphi}_{M}^{V}(0)\right|^{2}
$$

где мы, конечно, пренебрегли массой электрона. Эта классическая формула была впервые получена в работе [21] и затем в работе [22]. Если обратиться к конкретным векторным мезонам, то $e_{q}^{2}$ в случае $\rho$-мезона следует положить равным $\left(e_{u}^{2}-e_{d}^{2}\right) / \sqrt{2}=$ $1 /(3 \sqrt{2})$, в случае $J / \psi$-мезона $e_{c}^{2}=4 / 9$ и для $\Upsilon$-мезона $e_{b}^{2}=1 / 9$.

Для ширины распада $\pi$-мезона (64) в статическом приближении, очевидно, будем иметь

$$
\Gamma_{\pi^{-} \rightarrow e \bar{\nu}_{e}}=\frac{3 G^{2} m_{e}^{2}}{2 \pi}\left|\widetilde{\varphi}_{M}^{\pi}(0)\right|^{2} .
$$

Квазипотенциальный подход можно применить и еще к одному классическому распаду $\pi^{0} \rightarrow \gamma \gamma$, структура амплитуды которого в системе покоя имеет вид

$$
\mathcal{M}\left(\mathbf{p}_{1} \mid M\right)=4 \pi \alpha f_{0} i \epsilon_{i j k} e_{1}^{*_{i}} e_{2}^{*} p_{1}^{k},
$$

где $\mathbf{p}_{1}$ - импульс одного из фотонов, $\mathbf{e}_{1}$ и $\mathbf{e}_{2}$ - векторы поляризации фотонов, а индексы $i, j, k$ принимают значения $1,2,3$. В квазипотенциальном подходе формфактор этого распада дается формулой

$$
\begin{aligned}
& f_{0}=\frac{8 m_{q} e_{q}^{2}}{(2 \pi)^{3}} \times \\
& \times \int d^{3} \omega_{\mathbf{k}_{1}}\left[\sqrt{\left(\mathbf{p}_{1}-\mathbf{k}_{1}\right)^{2}+m_{q}^{2}}\left(\sqrt{\left(\mathbf{p}_{1}-\mathbf{k}_{1}\right)^{2}+m_{q}^{2}}+k_{1}^{0}-p_{1}^{0}-i 0\right)\right]^{-1} \varphi_{M}^{\pi}\left(\mathbf{k}_{1}\right),
\end{aligned}
$$


где, как и в случае $\rho$-мезона, $e_{q}^{2}=1 /(3 \sqrt{2})$. Нетрудно вычислить теперь ширину распада в статическом приближении,

$$
\Gamma_{\pi^{0} \rightarrow \gamma \gamma}=\frac{3 \pi \alpha^{2} M}{4} f_{0}^{2}=\frac{32 \pi \alpha^{2}}{3 M^{2}\left(z_{\pi}^{2}+1\right)\left(\sqrt{z_{\pi}^{2}+1}+z_{\pi}-1\right)^{2}}\left|\widetilde{\varphi}_{M}^{\pi}(0)\right|^{2},
$$

где параметр $z_{\pi}=2 m_{q} / M$.

Допуская теперь универсальность взаимодействия $u$ - и $d$-кварков, можно считать, что в формулах (72) и (75) стоит одна и та же волновая функция $\widetilde{\varphi}_{M}^{\pi}(0)$. В этом случае, исключая ее из обеих формул и используя экспериментальные значения для ширин распадов $\Gamma_{\pi^{-} \rightarrow e \bar{\nu}_{e}}$ и $\Gamma_{\pi^{0} \rightarrow \gamma \gamma}[23]$, нетрудно вычислить значение параметра $z_{\pi}$. Оно оказывается равным примерно 6 , т.е. имеется огромный дефект массы. Это означает, что взаимодействие кварков внутри мезона является сушественно релятивистским и применение статического приближения, во всяком случае, для этих легких мезонов неправомерно. Таким образом, в релятивистском случае необходимо знание волновых функций мезонов, которые являются решениями квазипотенциального уравнения, и подставляя которые в формулы (63), (68) и (74), можно вычислить соответствуюшие константы распада. Приложение квазипотенциального подхода к полулептонным распадам $B$-мезонов с использованием квазипотенциальных волновых функций можно найти, например, в работе [24].

\section{8. СТРУКТУРНЫЕ ФУНКЦИИ АДРОНОВ}

Квазипотенциальньй подход легко обобшается на случай взаимодействия трех и более частиц. Это позволило успешно применить его к описанию инклюзивных процессов и структурных функций адронов. Квазипотенциальное уравнение для системы $n$ частиц имеет вид [25]

$$
\begin{aligned}
& 2 \varepsilon_{k_{n}}\left(\varepsilon_{k_{1}}+\cdots+\varepsilon_{k_{n}}-\sqrt{s}\right) \Psi_{P}\left(\mathbf{k}_{1}, \ldots, \mathbf{k}_{n-1}\right)= \\
& \quad=\int d^{3} \omega_{\mathbf{k}_{1}^{\prime}} \ldots d^{3} \omega_{\mathbf{k}_{n-1}^{\prime}} V\left(\mathbf{k}_{1}, \ldots, \mathbf{k}_{n-1} ; \mathbf{k}_{1}^{\prime}, \ldots, \mathbf{k}_{n-1}^{\prime} \mid P, \sqrt{s}\right) \Psi_{P}\left(\mathbf{k}_{1}^{\prime}, \ldots, \mathbf{k}_{n-1}^{\prime}\right) .
\end{aligned}
$$

В квазипотенциальном подходе к описанию инклюзивных процессов предполагается, что адрон состоит из большого числа партонов (кварков), которые первоначально связаны внутри него, но в результате взаимодействия он "рассьпается" на составные части. При этом амплитуды инклюзивных реакций удается выразить через одновременные волновые функции, описывающие адроны как связанные состояния кварков, а также через амплитуды субпроцессов с участием кварков, глюонов и других частиц [26]. Наличие вероятностной интерпретации квазипотенциальных волновых функций позволяет построить из них плотности вероятности, имеющие смысл функций распределения импульсов кварков внутри адронов, т.е. структурных функций.

Так, при рассмотрении глубоконеупругого рассеяния лептона на адроне амплитуда испускания виртуального фотона, поглошаемого затем лептоном, может быть записана аналогично амплитуде распада (60) следуюшим образом:

$$
\begin{aligned}
& \mathcal{M}\left(q, \mathbf{k}_{1}, \ldots, \mathbf{k}_{n-1} \mid P, M\right)= \\
& =\int d^{3} \omega_{\mathbf{k}_{1}^{\prime}} \ldots d^{3} \omega_{\mathbf{k}_{n-1}^{\prime}} T\left(q, \mathbf{k}_{1}, \ldots, \mathbf{k}_{n-1} ; \mathbf{k}_{1}^{\prime}, \ldots, \mathbf{k}_{n-1}^{\prime} \mid P, M\right) \Psi_{P}\left(\mathbf{k}_{1}^{\prime}, \ldots, \mathbf{k}_{n-1}^{\prime}\right),
\end{aligned}
$$


где $T(P, M)$ - амплитуда радиационного перехода системы $n$ составляюших с испусканием виртуального фотона с 4-импульсом $q$. Как и при рассмотрении высокоэнергетического упругого рассеяния, мы здесь ограничимся случаем одинаковых частиц и пренебрежем спинами. Амплитуда $T(P, M)$ может быть представлена в виде суммы амплитуд взаимодействия виртуального фотона с каждым из партонов. Тогда, ограничиваясь лишь членами когерентного взаимодействия и принимая во внимание тождественность партонов, можно записать [27] вероятность излучения фотона в виде

$$
\begin{aligned}
& w\left(\nu, Q^{2}\right)=4 \pi \alpha n e_{c}^{2}(2 m)^{2} \times \\
& \times \int d^{3} \omega_{\mathbf{k}_{1}} \ldots d^{3} \omega_{\mathbf{k}_{n}}(2 \pi)^{4} \delta^{(4)}\left(q+k_{1}+\cdots+k_{n}-P\right)\left|\Psi_{P}\left(\mathbf{k}_{1}, \ldots, \mathbf{k}_{n-1}\right)\right|^{2},
\end{aligned}
$$

где $e_{c}$ - заряд партона, $\nu=\varepsilon_{q}$ и $Q^{2}=-q^{2}$. Сделав замену переменных $k_{n}=k-q$ и положив $\left(P-k_{1}-\cdots-k_{n-1}\right)^{2}=m^{2}$, получаем

$$
w\left(\nu, Q^{2}\right)=4 \pi \alpha n e_{c}^{2}(2 m)^{2} \int d^{3} \omega_{\mathbf{k}} 2 \pi \delta\left(2 k q-Q^{2}\right) I\left(\varepsilon_{k}\right),
$$

где

$$
\begin{aligned}
I\left(\varepsilon_{k}\right)= & \int d^{3} \omega_{\mathbf{k}_{1}} \ldots d^{3} \omega_{\mathbf{k}_{n-1}}(2 \pi)^{3} 2 k^{0} \times \\
& \times \delta^{(3)}\left(\mathbf{k}_{1}+\cdots+\mathbf{k}_{n-1}+\mathbf{k}-\mathbf{P}\right)\left|\Psi_{P}\left(\mathbf{k}_{1}, \ldots, \mathbf{k}_{n-1}\right)\right|^{2}
\end{aligned}
$$

- релятивистски-инвариантная функция, полностью определяемая квадратом квазипотенциальной волновой функции составного адрона.

Перейдем в систему покоя адрона $\mathbf{P}=0$. Тогда, проинтегрировав по сферическим углам в формуле (79), получим

$$
w\left(\nu, Q^{2}\right)=\frac{\alpha n e_{c}^{2}(2 m)^{2}}{2 \nu^{\prime}} \int_{k_{\min }}^{\infty} \frac{d k k}{k^{0}} I\left(k^{0}\right),
$$

где $\nu^{\prime}=\sqrt{\nu^{2}+Q^{2}}$ и, если пренебречь массами партонов, $2 k_{\min }=\nu^{\prime}-\nu$. Из условия нормировки волновой функции следует, что

$$
\frac{1}{2 \pi^{2}} \int_{0}^{\infty} d k k^{2} I\left(k^{0}\right)=2 M-\left\langle\frac{\partial V(P, M)}{\partial M}\right\rangle .
$$

В это выражение входит усредненная по волновым функциям производная от квазипотенциала по полной энергии системы.

Введем масштабную переменную Нахтмана $\xi=\left(\nu^{\prime}-\nu\right) / M$. Тогда интеграл в формуле (81) будет зависеть только от этой переменной, которая изменяется в следующих пределах:

$$
0 \leqslant \xi \leqslant \xi_{\max }=\frac{Q^{2}}{2 M^{2}}\left(\sqrt{1+\frac{4 M^{2}}{Q^{2}}}-1\right)
$$


Вводя структурную функцию адрона $F\left(\xi, Q^{2}\right)$, вероятность $(81)$ можно записать в виде

$$
w\left(\nu, Q^{2}\right)=\frac{8 \pi \alpha M}{\nu^{\prime}} F\left(\xi, Q^{2}\right),
$$

причем структурная функция нормирована таким образом, что при переходе к точечному адрону $F\left(\xi, Q^{2}\right)=2 \pi \xi \delta\left(\xi_{\max }-\xi\right)$. Сравнивая $(84)$ и $(81)$, получаем

$$
F\left(\xi, Q^{2}\right)=\frac{n e_{c}^{2} m^{2}}{4 \pi M} \int_{M \xi / 2}^{k_{\max }} d k I\left(k^{0}\right),
$$

где верхний предел интегрирования по $k$ введен из тех соображений, что структурная функция должна обращаться в нуль на пороге физической области, и поэтому следует выбрать $k_{\max }=M \xi_{\max } / 2$. Таким образом, структурная функция адрона зависит только от масштабной переменной $\xi$, если пренебречь ее слабым возрастанием с $Q^{2}$ за счет изменения верхнего предела интегрирования. Заметим, что в пределе больших $Q^{2}$, $Q^{2} \gg 4 M^{2}$, переменная $\xi$ совпадает с обычной переменной Бьеркена $x=Q^{2} / 2 M \nu$, которая изменяется строго на отрезке $[0,1]$.

Вычислим теперь моменты структурной функции (85). Переставляя порядок интегрирования, получаем

$$
M_{N-1}=\int_{0}^{\xi_{\max }} d \xi \xi^{N-1} F\left(\xi, Q^{2}\right)=\frac{n e_{c}^{2} m^{2}}{4 \pi N M}\left(\frac{2}{M}\right)^{N} \int_{0}^{k_{\max }} d k k^{N} I\left(k^{0}\right) .
$$

В частности, для первого момента в силу формулы (82) имеем

$$
M_{1}=\frac{\pi n e_{c}^{2} m^{2}}{M^{3}}\left[2 M-\left\langle\frac{\partial V(P, M)}{\partial M}\right\rangle\right] .
$$

Отметим, что в работах [26] в рамках квазипотенциального подхода подробно исследовались полуинклюзивные распределения, а также связь моментов полуинклюзивного распределения со структурными функциями.

\section{9. ЗАКЛЮЧЕНИЕ}

В данной работе рассмотрены некоторые следствия применения квазипотенциального подхода к релятивистским кварковым моделям адронов. Особое внимание уделено возможным методам построения квазипотенциала в каждой конкретной модели. Конечно, в одной журнальной статье невозможно охватить весь спектр результатов, полученных в рамках квазипотенциального подхода за 40 лет его существования. Однако, как нам кажется, уже рассмотренные здесь проблемы показьвают эффективность и последовательность такого подхода в физике релятивистских частиц.

Квазипотенциальный подход дает основу для теоретического описания релятивистских составных систем в рамках обших принципов и методов локальной квантовой теории поля. Важно, что при этом сохраняется преемственность с нерелятивистской квантовой механикой, поскольку основным объектом исследования здесь выступает волновая функция системы, удовлетворяющая определенному динамическому уравнению и физическим граничным условиям. 
Однако сам квазипотенциал, в отличие от классического потенциала, даже в случае чисто электромагнитного взаимодействия становится нелокальным, комплексным и явно зависящим от полной энергии системы. При достаточно малых импульсах частищ он может быть приближенно сведен к локальному и исследован в конфигурационном пространстве относительных расстояний между частищами. При этом оказывается, что при положительных энергиях связи квазипотенциал осциллирует, причем частота осциллящий определяется величиной энергии связи. В случае пренебрежимо малой энергии связи он переходит в обычный нерелятивистский кулоновский потенциал.

В работе кратко изложен квазипотенциальный подход в релятивистском конфигурационном пространстве, связанном с импульсным пространством преобразованием с помошью функций, реализующих представление группы движений в пространстве Лобачевского. При построении квазипотенциала взаимодействия двух адронов в конфигурационном пространстве сушественную роль играет гипотеза о гладкости локального квазипотенциала, которая отражает свойство протяженности и “рыхлости" адронов, участвующих в столкновении при высоких энергиях. Эта гипотеза позволила объяснить целый ряд основных закономерностей упругого рассеяния частиц высоких энергий на малые и большие углы.

Исследована задача взаимодействия частиц в релятивистском конфигурационном пространстве посредством эмпирического кулоновского квазипотенциала. Показано, что этот квазипотенциал моделирует свойство так называемой асимптотической свободы ( "бегущей" константы связи) в квантовой хромодинамике. Важным выводом является то, что спектр релятивистских связанных состояний в кулоновском квазипотенциале с большой константой связи сушественно отличается от нерелятивистского кулоновского спектра. При включении “запирающего" квазипотенциала для вычисления реалистических спектров масс и ширин распадов мезонов удается использовать квазиклассическое приближение. Релятивистский характер движения частиц сушественно меняет гранищы классически доступной области, а следовательно, и правила квантования.

Квазипотенциальный подход оказывается эффективным и для описания формфакторов распада мезонов. При этом, пожалуй, наиболее ярко проявляется необходимость учета релятивизма волновой функции составной системы, обладаюшей большим дефектом массы.

Квазипотенциальный подход легко обобщается на случай взаимодействия трех и более частиц. Здесь в качестве приложения общего метода мы ограничились рассмотрением глубоконеупругого рассеяния лептона на адроне с тем, чтобы продемонстрировать на простейшем примере, как наблюдаемые структурные функции мезона могут быть выражены через его волновую функцию.

\section{Список литературы}

[1] Н. Н. Боголюбов, А. А. Логунов, А. И. Оксак, И. Т. Тодоров. Общие принципы квантовой теории поля. М.: Наука, 1987.

[2] E. E. Salpeter, H. A. Bethe. Phys. Rev. 1951. V. 84. № 6. P. 1232.

[3] A. A. Logunov, A. N. Tavkhelidze. Nuovo Cimento. 1963. V. 29. № 2. P. 380.

[4] W. Macke. Z. Naturforsch. 1953. V. 8a. P. 599; W. Zimmermann. Nuovo Cimento. Suppl. 1954. V. 11. P. 1; W. Krolikowski, J. Rzewuski. Nuovo Cimento. 1955. V. 2. P. 203; 
R. Blankenbecler, R. Sugar. Single linear integral equation for relativistic scattering. In: Proc. 12th Annual Intern. Conf. on HEP. V. 1. Ed. Ya. A. Smorodinsky. M.: Atomizdat, 1966. P. 225.

[5] V. G. Kadyshevsky. Nucl. Phys. B. 1968. V. 6. № 2. P. 125; А. А. Логунов, В. И. Саврин, H. Е. Тюрин, О. А. Хрусталев. ТМФ. 1971. Т. 6. № 2. С. 157; R. N. Faustov. Ann. Phys. 1973. V. 78. № 1. Р. 176; А. А. Архипов, В. И. Саврин. ЭЧАЯ. 1985. Т. 16. № 5. С. 1091.

[6] П. Н. Боголюбов. О знаке мнимой части квазипотенциала. Препринт ОИЯИ Р2-5021. Дубна: ОИЯИ, 1970.

[7] A. A. Logunov, A. N. Tavkhelidze, I. T. Todorov, O. A. Khrustalev. Nuovo Cimento. 1963. V. 30. P. 134 .

[8] Р. Н. Фаустов. ЭЧАЯ. 1972. Т. 3. № 1. С. 238.

[9] Б.А. Арбузов, А.А. Логунов, А.Н. Тавхелидзе, Р.Н. Фаустов, А. Т. филиппов. ЖЭТФ. 1963. Т. 44. С. 1409; Б. А. Арбузов, А. А. Логунов, А. Т. Филиппов, О. А. Хрусталев. ЖЭТФ. 1964. Т. 46. С. 1266.

[10] В. Н. Капшай, В. И. Саврин, Н. Б. Скачков. ТМФ. 1986. Т. 69. № 3. С. 400.

[11] Б. А. Арбузов, Э. Э. Боос, В. И. Саврин, С. А. Шичанин. Письма в ЖЭТФ. 1989. Т. 50. № 5. C. 236 .

[12] V. G. Kadyshevsky, R. M. Mir-Kasimov, N. B. Skachkov. Nuovo Cimento. A. 1968. V. 55. № 2. Р. 233 ; В. Г. Кадышевский, Р. М. Мир-Касимов, Н. Б. Скачков. ЭЧАЯ. 1972. Т. 2. № 3. C. 635 .

[13] S. P. Alliluyev, S. S. Gershtein, A. A. Logunov. Phys. Lett. 1965. V. 18. № 2. P. 195.

[14] В.Р. Гарсеванишвили, В. А. Матвеев, Л. А. Слепченко. ЭЧАЯ. 1970. Т. 1. № 1. С. 91; V.R. Garsevanishvili, V.A. Matveev, L. A. Slepchenko, A. N. Tavkhelidze. Phys. Rev. D. 1971. V. 1. № 4. P. 849 .

[15] А. А. Логунов, О. А. Хрусталев. ЭЧАЯ. 1970. Т. 1. № 1. С. 71.

[16] V. A. Novikov, L. B. Okun, M. A. Shifman, A. I. Vainshtein, M. V. Voloshin, V. I. Zakharov. Phys. Rep. C. 1978. V. 41. № 1. P. 1.

[17] V. I. Savrin, N. B. Skachkov. Nuovo Cimento Lett. 1980. V. 29. № 11. P. 363.

[18] А. Д. Донков, В. Г. Кадьиевский, М. Д. Матеев. ТМФ. 1982. Т. 50. № 3. С. 360.

[19] V. I. Savrin, A. V. Sidorov, N. B. Skachkov. Hadronic J. 1981. V. 4. № 5. P. 1642.

[20] В. И. Саврин, Н. Б. Скачков. Формфакторы и структурные функции адронов в одновременной формулировке квантовой теории поля. В сб.: Проблемы физики высоких энергий и теории поля (V международный семинар). Протвино: ИФВЭ, 1982. Т. II. С. 229.

[21] В. А. Матвеев, Б. В. Струминский, А. Н. Тавхелидзе. Некоторые эффекты в модели кварков. Сообщение ОИЯИ Р-252. Дубна: ОИЯИ, 1965.

[22] R. Van Royen, V. F. Weisskopf. Nuovo Cimento. A. 1967. V. 50. № 3. P. 617.

[23] D. E. Groom et al. (Particle Data Group). Europ. Phys. J. 2000. V. C15. P. 1

[24] R. N. Faustov, V. O. Galkin, A. Yu. Mishurov. Phys. Lett. B. 1995. V. 536. P. 516.

[25] B. А. Матвеев, Р. М. Мурадян, А. Н. Тавхелидзе. Релятивистски ковариантные волновые уравнения для $N$ частиц в квантовой теории поля. Препринт ОИЯИ Р2-3900. Дубна: ОИЯИ, 1968; А. А. Архипов, В. И. Саврин. ТМФ. 1973. Т. 16. № 3. С. 328.

[26] В. А. Матвеев, А. Н. Сисакян, Л. А. Слепченко. ЯФ. 1976. Т. 23. № 2. С. 432; А. Н. Квинихидзе, А.Н. Сисакян, Л.А. Слепченко, А.Н. Тавхелидзе. ЭЧАЯ. 1977. V. 8. № 3. P. 478.

[27] М. Н. Дубинин, В. И. Саврин, Н. Б. Скачков. ЯФ. 1990. Т. 52. № 5(11). С. 1273. 